\title{
Warm pleasant feelings in the brain
}

\author{
Edmund T. Rolls, ${ }^{*}$ Fabian Grabenhorst, and Benjamin A. Parris \\ University of Oxford, Department of Experimental Psychology, South Parks Road, Oxford OX1 3UD, England, UK
}

Received 2 January 2008; revised 26 February 2008; accepted 7 March 2008

Available online 18 March 2008

\begin{abstract}
Warm and cold stimuli have affective components such as feeling pleasant or unpleasant, and these components may have survival value, for approach to warmth and avoidance of cold may be reinforcers or goals for action built into us during evolution to direct our behaviour to stimuli that are appropriate for survival. Understanding the brain processing that underlies these prototypical reinforcers provides a direct approach to understanding the brain mechanisms of emotion. In an fMRI investigation in humans, we showed that the mid-orbitofrontal and pregenual cingulate cortex and the ventral striatum have activations that are correlated with the subjective pleasantness ratings made to warm $\left(41^{\circ} \mathrm{C}\right)$ and cold $\left(12^{\circ} \mathrm{C}\right)$ stimuli, and combinations of warm and cold stimuli, applied to the hand. Activations in the lateral and some more anterior parts of the orbitofrontal cortex were correlated with the unpleasantness of the stimuli. In contrast, activations in the somatosensory cortex and ventral posterior insula were correlated with the intensity but not the pleasantness of the thermal stimuli. A principle thus appears to be that processing related to the affective value and associated subjective emotional experience of thermal stimuli that are important for survival is performed in different brain areas to those where activations are related to sensory properties of the stimuli such as their intensity. This conclusion appears to be the case for processing in a number of sensory modalities, and the finding with such prototypical stimuli as warm and cold provides strong support for this principle.

(C) 2008 Elsevier Inc. All rights reserved.
\end{abstract}

Keywords: Emotion; Affect; Warmth; Temperature; fMRI; Humans

\section{Introduction}

Warm and cold stimuli have affective components such as feeling pleasant or unpleasant, and these components may have survival value, for approach to warmth and avoidance of cold may be reinforcers or goals for action built into us during evolution to direct

\footnotetext{
* Corresponding author. Fax: +44 1865310447.

E-mail address: Edmund.Rolls@oxcns.org (E.T. Rolls).

URL: http://www.oxcns.org (E.T. Rolls).
}

Available online on ScienceDirect (www.sciencedirect.com). our behaviour to stimuli that are important for survival (Rolls, 2005). Indeed, warm and cold stimuli may be important prototypical primary, that is unlearned, reinforcers, and investigation of the neural mechanisms that are related to these stimuli and the feelings they arouse may provide a direct approach to understanding the brain mechanisms of emotion and indeed of decision-making (Cabanac, 2002; Rolls, 1999, 2005, 2008b). The aim of this functional magnetic resonance imaging (fMRI) investigation was to determine whether there are special brain areas that process and make explicit in the representation the affective attributes of warm and cold thermal stimuli, as compared to other sensory attributes of thermal stimuli, such as their intensity. In addition, we tested the hypothesis of whether different brain regions track the subjective feelings of pleasantness vs unpleasantness evoked by thermal stimuli. Warm and cool thermal stimuli, and mixtures of them, were applied to the hand. The design allowed us not only to investigate where warm and cold stimuli are represented, but also how the brain systems that represent their affective value respond to mixtures of the warm and cold stimuli. Representation of affective value may be important as a preliminary process to making decisions about whether to treat the mixed stimuli as rewards or punishers. Because the human participants made ratings of the pleasantness and intensity of the stimuli on every trial during the fMRI investigation, we were able to analyse how their subjective feelings of pleasantness, unpleasantness, and intensity were related to activations in different brain regions. We explicitly did not use in this study thermal stimuli that were painful, whether hot or cold, but restricted the temperatures applied for $5 \mathrm{~s}$ at a time to the hand to the range $12-41^{\circ} \mathrm{C}$, with values selected individually for each subject to be rated as cold and unpleasant but not painful, or warm and pleasant.

There have been very few previous investigations of the affective representation in the human brain of non-noxious (non-painful) thermal stimuli including mixtures of thermal stimuli, and one of the new aims of this study was to investigate affective but non-noxious thermal representations in the brain, and relate these to subjective ratings of pleasantness and unpleasantness. For example, painful hot and cold stimuli activate brain regions including the posterior insula, cingulate cortex, and somatosensory, premotor and motor areas (Brooks et al., 2005; Craig et al., 2000, 1996; Tracey et al., 2000). Becerra et al. (2001) showed considerable overlap between brain 
areas activated by a noxious hot stimulus $\left(46^{\circ} \mathrm{C}\right)$ and a warm stimulus $\left(41{ }^{\circ} \mathrm{C}\right)$, and did not use correlation analyses with pleasant and unpleasant subjective ratings to investigate whether different brain regions process affectively pleasant vs affectively unpleasant nonnoxious thermal stimuli. In a PET study, activations in the insula and part of the orbitofrontal cortex were related to the perceived intensity of cool stimuli, but affective value (pleasantness and unpleasantness) ratings were not obtained, and warm stimuli were not used (Craig et al., 2000). In another PET study, activations to warmth were found in the orbitofrontal cortex, but no potential correlations with pleasantness ratings were investigated (Casey et al., 1996). At the neuronal level, it has been shown that non-noxious oral thermal stimuli activate single neurons in the orbitofrontal cortex (Kadohisa et al., 2004), amygdala (Kadohisa et al., 2005b), and insular taste cortex (Verhagen et al., 2004), with different neurons having increasing, decreasing, or tuned responses as a function of the temperature. Moreover, some of these neurons are thermo-specific, and others combine thermal sensitivity with responses to taste, and/or oral texture (including viscosity and/or fat texture) (Kadohisa et al., 2004, 2005a,b; Verhagen et al., 2004). In a related study, it was shown in humans that oral temperature stimuli can activate the insular taste cortex, the orbitofrontal and pregenual cingulate cortex, and the somatosensory cortex (Guest et al., 2007), but stimulation of the external body surface was not investigated, nor were mixtures of thermal stimuli investigated. The novel aims of the present study were to investigate the representation of affective but non-noxious thermal stimuli, and mixtures of them, in the brain, and to relate these to subjective ratings of the pleasantness, unpleasantness and intensity of the stimuli.

\section{Methods}

\section{Design}

In the experiment described here, we compared brain responses to a warm pleasant stimulus $\left(41^{\circ} \mathrm{C}\right)$ applied to the hand ("warm2"), a cool unpleasant stimulus $\left(12{ }^{\circ} \mathrm{C}\right)$ applied to the hand ("cold"), a combined warm and cold stimulus ("warm $2+$ cold"), and a second combination designed to be less pleasant $\left(39^{\circ} \mathrm{C}+12^{\circ} \mathrm{C}\right)($ "warm $1+$ cold"). The stimuli were delivered in random permuted sequence, and on every trial the participant rated the subjective pleasantness and subjective intensity of the stimulus. Two ratings of pleasantness were taken, one for values in the range 0 (neutral) to +2 (very pleasant), and the second for values in the range 0 to -2 (very unpleasant), to investigate whether the activations in similar brain areas were correlated with the pleasantness of stimuli both when they were pleasant $(\geq 0)$, and when they were unpleasant $(\leq 0)$, or whether different brain areas code for thermal stimuli that are pleasant or unpleasant.

We investigated how the thermal component stimuli and the mixtures were represented in brain areas identified by prior hypotheses such as the orbitofrontal and anterior cingulate cortex and ventral striatum where the pleasantness and unpleasantness of touch and oral temperature are represented (Guest et al., 2007; Rolls et al., 2003b), and in the insula and somatosensory cortex where thermal stimuli are represented (Brooks et al., 2005; Craig et al., 2000, 1996; Tracey et al., 2000). Given the aims of the investigation, we used both SPM (Wellcome Institute of Cognitive Neurology) correlation analyses between the subjective ratings and the activations in these brain areas, and SPM contrasts between the activations produced to the different thermal stimuli, in these brain areas, to investigate the effects of the thermal stimuli.

\section{Participants}

Twelve healthy volunteers (6 male and 6 female, mean age 26) participated in the study. Ethical approval (Central Oxford Research Ethics Committee) and written informed consent from all subjects were obtained before the experiment.

\section{Stimuli}

Controlled cool thermal stimuli were applied using an adapted commercially available Peltier thermode (MEDOC, Haifa, Israel; $30 \times 30 \mathrm{~mm}$ thermo-conducting surface) strapped to the dorsum of the left hand. The thermode produces a trapezoid-like stimulus, with a time to reach the target temperature of $12{ }^{\circ} \mathrm{C}$ of $5 \mathrm{~s}$, with a similar period to return to baseline temperature. The plateau temperature was held for $4 \mathrm{~s}$, and subsequent data analyses focused on brain activation during the time of this maintained (plateau) temperature. The warm stimulus was applied using a thermal resistor $20 \times 15 \mathrm{~mm}$ strapped to the palm of the left hand. The thermal resistor device was designed and built at the Oxford Centre for Functional Magnetic Resonance Imaging of the Brain (FMRIB), and ramped the temperature to $41{ }^{\circ} \mathrm{C}$ (for the "warm2" stimulus) or to $39^{\circ} \mathrm{C}$ (for the "warm1" stimulus) in $<2 \mathrm{~s}$ (Bantick et al., 2002). The placement of the stimuli on the dorsum and palm of the hand was designed to minimize thermal interaction between the stimuli in the short delivery period of $4 \mathrm{~s}$, and was designed so that even with any topologically mapped representation of the body surface that might be present in the activated brain regions, the regions of activation would be close in the brain. The method of stimulus delivery ensured that the devices were continually in place during the experiment, and that only temperature changes were occurring in the stimulation periods. In preliminary testing, the exact temperatures used for each subject were tailored $\pm 2^{\circ} \mathrm{C}$, so that warm 2 was rated as very pleasant; cold as unpleasant but not painful or very unpleasant so that when it was combined with warm 2 the combination sometimes at least was more pleasant than neutral; and warm1 was adjusted so that it was less pleasant than warm 2 and more pleasant than neutral.

\section{Experimental protocol}

During the fMRI experiment the subjects gave psychophysical ratings of pleasantness and intensity on every trial, so that correlation analyses between the ratings and the brain activations could be performed. The experimental protocol consisted of an event-related interleaved design presenting in random permuted sequence the four experimental conditions described above. Each trial started at time $0 \mathrm{~s}$ with a small $1 \mathrm{~s}$ visual stimulus to indicate the start of the trial, and at the same time, the thermal stimulus was switched on to allow it to reach plateau. The plateau was reached by time $=5 \mathrm{~s}$, and then a $1 \mathrm{~s}$ stimulus appeared on the visual display stating "Rate" to indicate that subjective ratings were needed on this trial. There was then a $4 \mathrm{~s}$ period in which the temperature stimuli were held constant and a green cross was shown indicating to the subject that this was the relevant period for which ratings were required. It was made clear to the subjects in the instructions that this was the steady state period within which the evaluation of the pleasantness and intensity of the stimuli was to be determined by them. The actual ratings were made later, as described next, so that no aspect of making the ratings would occur in the steady state period in which the stimuli were being evaluated. After the $4 \mathrm{~s}$ 
plateau period, the thermal stimuli were switched off. The subjective ratings were then made. The first rating was for the pleasantness of the stimulus in the plateau period for values of 0 (neutral) to +2 (very pleasant). The second rating was for the pleasantness of the stimulus in the plateau period for values of 0 (neutral) to -2 (very unpleasant). The third rating was for the intensity of the stimulus in the plateau period on a scale from 0 (very weak) to 4 (very intense). The ratings were made with a visual analogue rating scale in which the subject moved the bar to the appropriate point on the scale using a button box. Subjects were pre-trained outside the scanner in the whole procedure and use of the rating scales. Each of the four trial types was presented in random permuted sequence 15 times. This general protocol and design has been used successfully in previous studies to investigate activations and their relation to subjective ratings in cortical areas (de Araujo et al., 2005; Grabenhorst et al., 2007a,b; Rolls et al., 2003a,b). On some other trials, not included in the experiment described here, instead of "Rate" the word "Decide" appeared, and the subjects had to decide whether they would choose to repeat the particular stimulus that had just been delivered if the opportunity was available for this after the experiment.

\section{fMRI data acquisition}

Images were acquired with a 3.0-T VARIAN/SIEMENS wholebody scanner at the Centre for Functional Magnetic Resonance Imaging at Oxford (FMRIB), where $27 \mathrm{~T} 2 *$ weighted EPI coronal slices with in-plane resolution of $3 \times 3 \mathrm{~mm}$ and between plane spacing of $4 \mathrm{~mm}$ were acquired every $2 \mathrm{~s}(\mathrm{TR}=2)$. We used the techniques that we have developed over a number of years (de Araujo et al., 2003; O'Doherty et al., 2001b) and as described in detail by Wilson et al. (2002) we carefully selected the imaging parameters in order to minimise susceptibility and distortion artefact in the orbitofrontal cortex. The relevant factors include imaging in the coronal plane, minimizing voxel size in the plane of the imaging, as high a gradient switching frequency as possible $(960 \mathrm{~Hz})$, a short echo time of $28 \mathrm{~ms}$, and local shimming for the inferior frontal area. The matrix size was $64 \times 64$ and the field of view was $192 \times 192 \mathrm{~mm}$. Continuous coverage was obtained from $+62(\mathrm{~A} / \mathrm{P})$ to $-46(\mathrm{~A} / \mathrm{P})$. A whole brain $\mathrm{T} 2 *$ weighted EPI volume of the above dimensions, and an anatomical T1 volume with coronal plane slice thickness $3 \mathrm{~mm}$ and in-plane resolution of $1 \times 1 \mathrm{~mm}$ was also acquired.

\section{fMRI data analysis}

The imaging data were analysed using SPM5 (Wellcome Institute of Cognitive Neurology). Pre-processing of the data used SPM5 realignment, reslicing with sinc interpolation, normalisation to the MNI coordinate system (Montreal Neurological Institute) (Collins et al., 1994), and spatial smoothing with a $6 \mathrm{~mm}$ full width at half maximum isotropic Gaussian kernel. The time series at each voxel were low-pass filtered with a haemodynamic response kernel. Time series non-sphericity at each voxel was estimated and corrected for (Friston et al., 2002), and a high-pass filter with a cut-off period of $128 \mathrm{~s}$ was applied. In the single event design, a general linear model was then applied to the time course of activation where stimulus onsets ( $t=0$ in each trial) were modelled as single impulse response functions and then convolved with the canonical haemodynamic response function (Friston et al., 1994). Linear contrasts were defined to test specific effects. Time derivatives were included in the basis functions set. Following smoothness estimation (Kiebel et al., 1999), linear contrasts of parameter estimates were defined to test the specific effects of each condition with each individual's dataset. Voxel values for each contrast resulted in a statistical parametric map of the corresponding $t$-statistic, which was then transformed into the unit normal distribution (SPM Z). The statistical parametric maps from each individual's dataset were then entered into second-level (group), random effects analyses accounting for both scan-to-scan and subject-to-subject variability. More precisely, the sets of individual statistical maps corresponding to a specific effect of interest were entered as covariates in multiple regression models as implemented in SPM5, and the corresponding group effects were assessed by applying linear contrasts (again following smoothness estimation) to the (second-level) parameter estimates generating a $t$-statistics map for each group effect of interest. The correlation analyses of the fMRI BOLD (blood oxygenation-level dependent) signal with given parameters of interest (e.g. the pleasantness ratings) were performed at the second-level through applying onesample $t$-tests to the first-level statistical parametric maps resulting from performing linear parametric modulation as implemented in SPM5. We report results for brain regions where there were prior hypotheses as described in the Methods, Design section, and applied small volume (false discovery rate) corrections for multiple comparisons (Worsley et al., 1996) with a radius corresponding to the full width at half maximum of the spatial smoothing filter used. Peaks are reported corrected for false discovery rate, with the exact corrected probability values (Worsley et al., 1996) given in the text.

To show the change in the \% BOLD signal, we located activations within the a priori regions of interest and extracted from the fitted time course the event-related responses from the peak voxel for each subject. These peaks of the single-subject time courses were then averaged across subjects.

For voxels where significant correlations were found between the $\%$ BOLD signal and the ratings, we produced graphs to show how the ratings were related to the \% BOLD signal. These were produced for each subject by taking the average of the BOLD response in the 3 time bins at 4,6 and 8s poststimulus, on each trial, and the corresponding rating. For each subject the means were calculated in discretized ranges of the rating function (e.g. -2 to $-1.75,-1.75$ to -1.5 etc), and then these values were averaged across subjects.

\section{Results}

\section{The ratings of pleasantness and intensity}

The ratings of the pleasantness and intensity of the stimuli obtained during the neuroimaging are shown in Fig. 1. It is clear that the warm2 stimulus was the most pleasant, the cold stimulus was the most unpleasant, and that the two mixture stimuli were placed in between, with the combination of warm $2+$ cold being more pleasant than the combination warm $1+$ cold. This is confirmed for the data shown in Fig. 1 (top) for the pleasantness ratings $>0$ by a within subjects ANOVA performed on the four thermal stimuli $\left(F(3,33)=54.6, p<10^{-7}\right)$ followed by post hoc LSD tests (accompanied by a KolmogorovSmirnov test for normality) showing that warm 2 was significantly different from the other stimuli $(p<0.001$ in all cases), and that warm $2+$ cold was significantly different from all other stimuli $(p<$ 0.02 in all cases). The same pattern is confirmed for the data shown in Fig. 1 (middle) for the pleasantness ratings $<0$ by within subjects ANOVA performed on the four thermal stimuli $(F(3,30)=33.8$, $p<10^{-6}$ ) followed by post hoc LSD tests (accompanied by a 

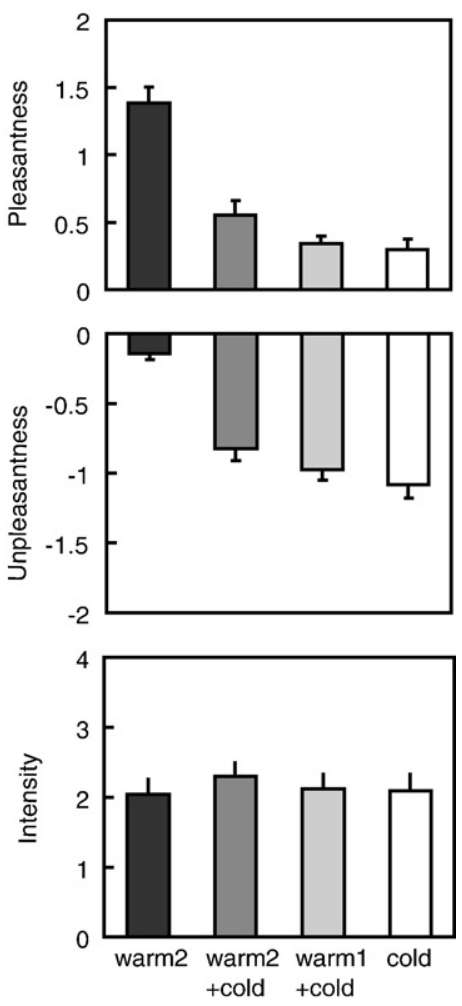

Fig. 1. The ratings of pleasantness and intensity for the stimuli (means \pm sem). Top: pleasantness ratings using the scale from 0 (neutral) to +2 (very pleasant). Middle: pleasantness ratings using the scale from 0 (neutral) to -2 (very unpleasant). Bottom: intensity ratings using the scale from 0 (very weak) to 4 (very intense).

Kolmogorov-Smirnov test for normality) showing that warm 2 was significantly different from the other stimuli $(p<0.001$ in all cases), and that warm2cold was significantly different from the warm2 stimulus $(p<0.001)$. There were no significant differences in the mean intensity ratings of the four stimuli $(F(3,33)=1.9, p=0.14)$.

\section{Activations related to warm pleasantfeelings in the mid-orbitofrontal cortex}

A correlation was found in the mid-orbitofrontal cortex at $[-26$ $38-10] z=3.25 p<0.04$ between the BOLD signal and the pleasantness ratings of the four thermal stimuli, as shown in Fig. 2a using the SPM analysis with pleasantness ratings as a regressor. To show how the rating values are related to the BOLD signal, we show in Fig. $2 b$ the \% BOLD values that correspond to the different values given for the pleasantness ratings on each trial (see Methods). There was a positive correlation for the data in Fig. $2 b$ between the subjective pleasantness ratings for positive values of the ratings and the BOLD signal $(r=0.84, p<0.01)$. A positive correlation between the subjective pleasantness ratings for negative values of the ratings and the BOLD signal was also found (Fig. 2c) ( $r=0.83, p=0.012$ ), showing that the activations in this brain region are related to the degree of pleasantness of the stimuli, for stimuli that had pleasantness ratings greater than zero, and less than zero. The activations in this part of the brain thus reflect the pleasantness of both pleasant and unpleasant thermal stimuli. The activations in this mid-orbitofrontal cortex region were not correlated with the intensity as shown by no significant correlation in the SPM correlation analysis, and by the lack of correlation shown in Fig. $2 \mathrm{~d}(r=0.07, p=0.8)$. To further elucidate the nature of the activation produced by the thermal stimuli in this region, Fig. 2e shows the peak values $( \pm$ sem $)$ of the \% BOLD signal change at this site for the four thermal stimuli. It can be seen that the BOLD signals are clearly graded in the order warm $2>$ warm $2+$ cold $>$ warm $1+$ cold $>$ cold, with activation to the warm 2 stimulus, and graded deactivation to the stimuli with cold. a
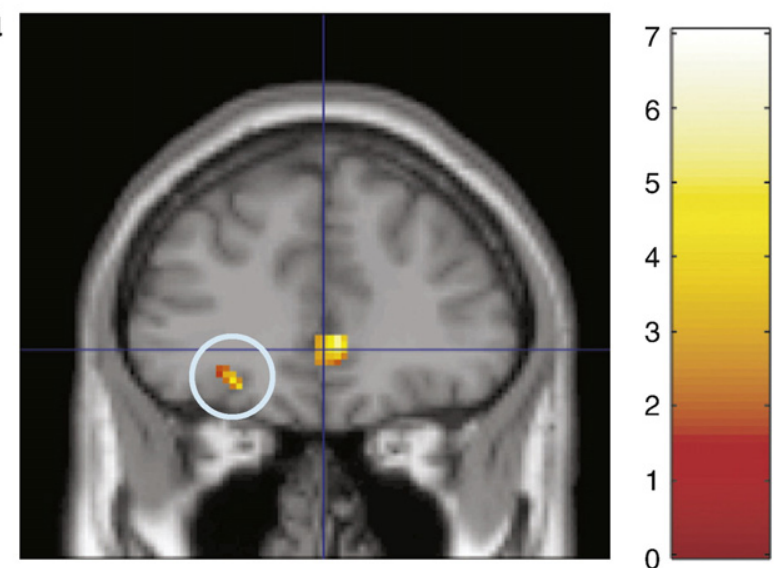

b

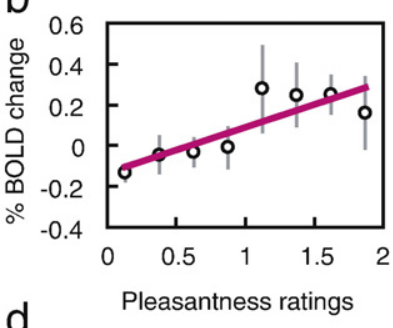

d

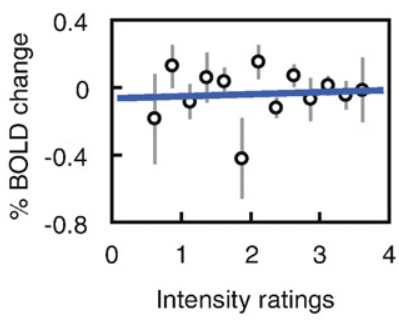

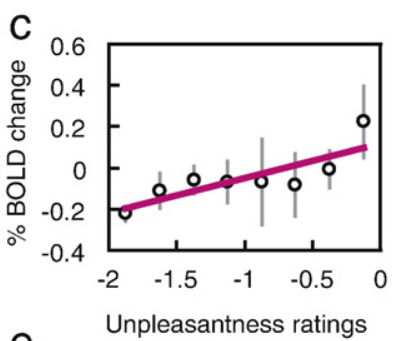

e

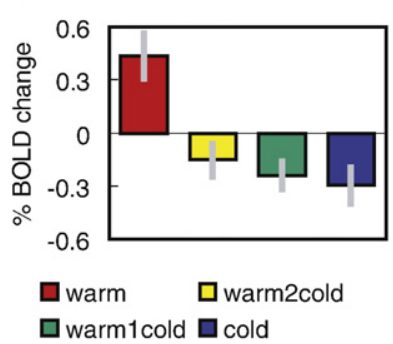

Fig. 2. Activations correlating with pleasantness in the orbitofrontal cortex. a. SPM analysis showing a correlation in the mid-orbitofrontal cortex (blue

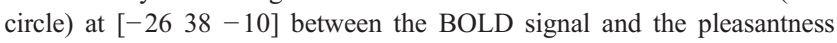
ratings of the four thermal stimuli. (Activations are also shown in the pregenual cingulate cortex in this coronal slice, and these are further illustrated in Fig. 3.) For this mid-orbitofrontal cortex region, (b) shows the positive correlation between the subjective pleasantness ratings for positive values of the ratings and the BOLD signal $(r=0.84, d f=7, p<0.01)$ (c) shows the positive correlation between the subjective pleasantness ratings for negative values of the ratings and the BOLD signal $(r=0.83, d f=7$, $p=0.012$ ); (d) shows that there is no correlation between the subjective intensity ratings and the BOLD signal $(r=0.07, d f=12, p=0.8)$; (e) shows the peak values $( \pm$ sem $)$ of the $\%$ BOLD signal change at this site for the 4 thermal stimuli. (The \% BOLD values in $b-d$ were calculated by obtaining the average $( \pm \mathrm{sem})$ BOLD signal for pleasantness or intensity ratings binned at increments of 0.25 for each subject, and then averaging across subjects.) 
Activations related to warm pleasant feelings in the pregenual cingulate cortex

The SPM analysis showed a correlation in the pregenual cingulate cortex at $[438-2] \mathrm{z}=4.24 p=0.001$ between the BOLD signal and the pleasantness ratings of the four thermal stimuli, as shown in Fig. 3a. A positive correlation between the subjective pleasantness ratings for positive values of the ratings and the BOLD signal was found $(r=0.92$, $p=0.001$ ), as shown in Fig. 3b. A non-significant (ns) positive correlation between the subjective pleasantness ratings for negative values of the ratings and the BOLD signal was found (Fig. 3c) $(r=0.66, p=0.07 \mathrm{~ns})$. The activations in this part of the brain thus reflect the pleasantness of both pleasant and unpleasant thermal stimuli. The activations in this pregenual cingulate cortex region had only a weak correlation with the intensity ratings as shown by a non-
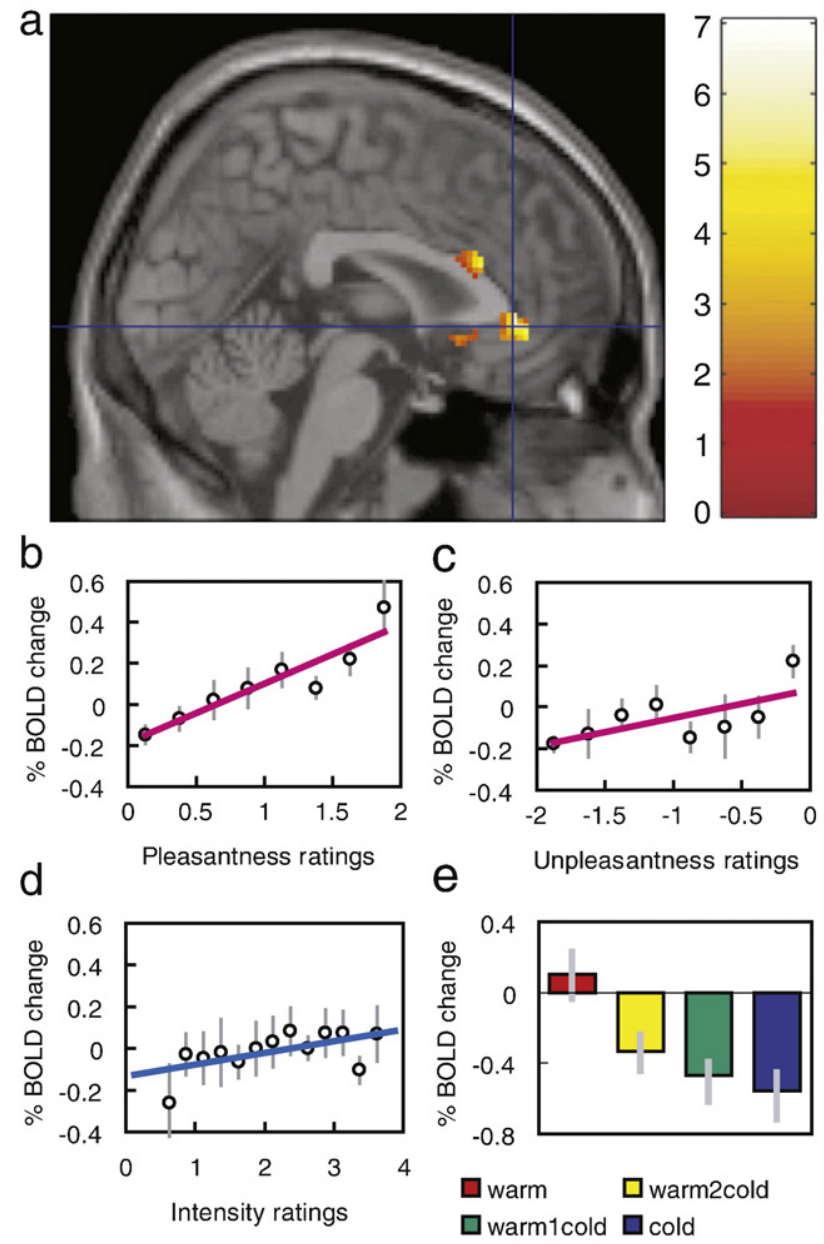

Fig. 3. Activations correlating with pleasantness in the pregenual cingulate cortex. a. SPM analysis showing a correlation in the pregenual cingulate cortex with peak at [4 38 -2] between the BOLD signal and the pleasantness ratings of the four thermal stimuli. For this pregenual cingulate cortex region, (b) shows the positive correlation between the subjective pleasantness ratings for positive values of the ratings and the BOLD signal ( $r=0.92$, $d f=7, p=0.001$ ) (c) shows the positive correlation between the subjective pleasantness ratings for negative values of the ratings and the BOLD signal ( $r=0.66, d f=7, p=0.07 \mathrm{~ns}$ ); (d) shows that there is only a low correlation between the subjective intensity ratings and the BOLD signal $(r=0.58$, $d f=12, p=0.037)$; (e) shows the peak values $( \pm$ sem $)$ of the $\%$ BOLD signal change at this site for the 4 thermal stimuli. significant correlation in the SPM correlation analysis, and by the small correlation shown in Fig. $3 \mathrm{~d}(r=0.58, \mathrm{p}<0.04)$. To further elucidate the nature of the activation produced by the thermal stimuli in this region, Fig. $3 e$ shows the peak values $( \pm$ sem $)$ of the $\%$ BOLD signal change at this site for the 4 thermal stimuli. It can be seen that the BOLD signals are graded in the order warm $2>$ warm $2+$ cold $>$ warm $1+$ cold $>$ cold, with activation to the warm 2 stimulus, and graded deactivation to the stimuli with cold. Indeed, in contrast analyses, this brain region showed significantly greater activation to warm 2 than cold $[640-6] z=2.98 p<0.04$.

In addition to this pregenual cingulate site, a somewhat more dorsal anterior cingulate site, evident in Fig. 3a, also had activations that were positively correlated with the pleasantness ratings of the thermal stimuli [ 6222 20] $z=4.04 p=0.002$. Indeed, in contrast analyses, this brain region showed significantly greater activation to warm2 than cold [ $\left[\begin{array}{lll}6 & 24 & 20\end{array}\right] z=4.14 p<0.001$.

\section{Activations related to warm pleasant feelings in the ventral striatum}

The SPM analysis showed a correlation in the ventral striatum at $\left[\begin{array}{lll}-2 & 20 & -4\end{array}\right] z=3.25 p=0.04$ between the BOLD signal and the pleasantness ratings of the four thermal stimuli, as shown in Fig. 4a. A positive correlation between the subjective pleasantness ratings for positive values of the ratings and the BOLD signal was found $(r=0.98, p<0.001)$, as shown in Fig. 4b. A positive correlation between the subjective pleasantness ratings for negative values of the ratings and the BOLD signal was also found (Fig. 4c) $(r=0.81$, $p=0.015)$. The activations in this part of the brain thus reflect the pleasantness of both pleasant and unpleasant thermal stimuli. The activations in this ventral striatal region were not correlated with the intensity ratings as shown by no significant correlation in the SPM correlation analysis, and by the lack of correlation shown in Fig. 4d $(r=0.28, p=0.36)$. To further elucidate the nature of the activation produced by the thermal stimuli in this region, Fig. 4e shows the peak values $( \pm$ sem) of the \% BOLD signal change at this site for the 4 thermal stimuli. It can be seen that the BOLD signals are graded in the order warm $2>$ warm $2+$ cold $>$ warm $1+$ cold $>$ cold, with activation to the warm 2 stimulus, and graded deactivation to the stimuli with cold. Indeed, in contrast analyses, this brain region showed significantly greater activation to warm 2 than cold $[-620-6] z=3.12 p=0.022$. Although the peak of this ventral striatal activation was found at $[-2$ $20-4]$, the activation extended clearly to $y=9 \mathrm{~mm}$ into the main part of the ventral striatum, and did not extend anteriorly.

A more lateral part of the ventral striatum had correlations with intensity but not pleasantness [ $\left.\begin{array}{lll}-10 & 10 & -14\end{array}\right] z=2.87 p=0.039$ (see Supplementary Material).

\section{Activations related to cold unpleasant feelings in the lateral orbitofrontal cortex}

The SPM analysis showed a negative correlation in the lateral orbitofrontal cortex with peak at $[-3840-6] z=3.42 p=0.032$ between the BOLD signal and the pleasantness ratings of the four thermal stimuli, as shown in Fig. 5a. A negative correlation between the subjective pleasantness ratings for positive values of the ratings and the BOLD signal was found ( $r=-0.86, p=0.006)$, as shown in Fig. 5b. A negative correlation between the subjective pleasantness ratings for negative values of the ratings and the BOLD signal was also found (Fig. 5c) $(r=-0.90, p=0.003)$. The activations in this part of the brain thus reflect the unpleasantness of both pleasant and unpleasant thermal stimuli. The activations in this lateral orbito- 
a

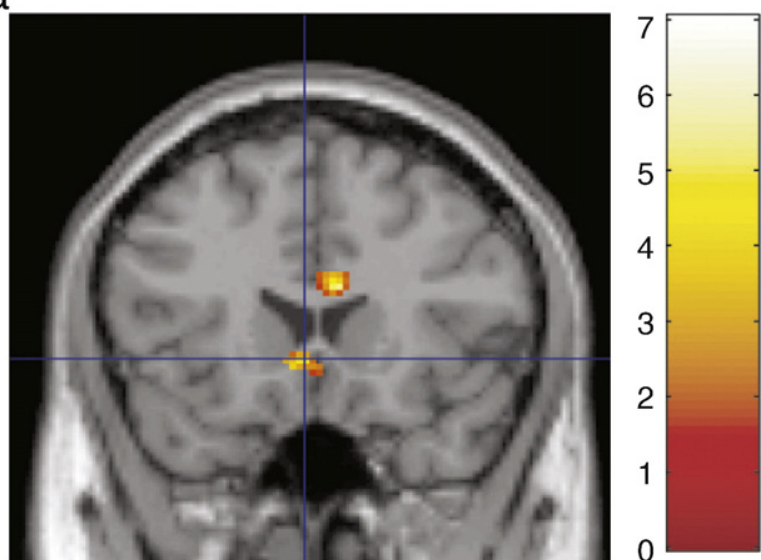

b

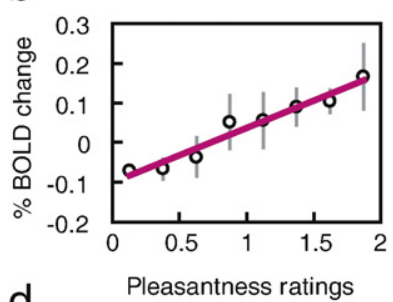

d

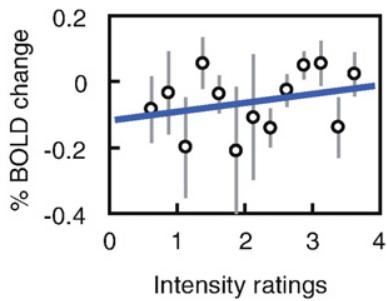

C

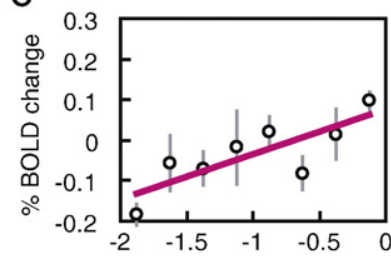

e

Unpleasantness ratings

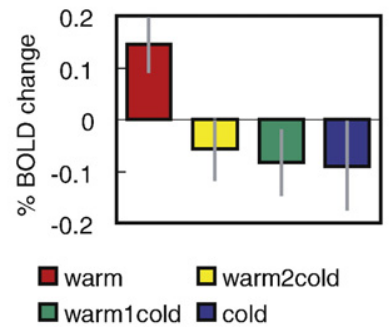

Fig. 4. Activations correlating with pleasantness in the ventral striatum. a. SPM analysis showing a correlation in the ventral striatum with peak at [ -220 -4 ] between the BOLD signal and the pleasantness ratings of the four thermal stimuli. For this ventral striatal region, (b) shows the positive correlation between the subjective pleasantness ratings for positive values of the ratings and the BOLD signal $(r=0.98, d f=7, p<0.001)$ (c) shows the positive correlation between the subjective pleasantness ratings for negative values of the ratings and the BOLD signal ( $r=0.81, d f=7, p=0.015)$; (d) shows that there is little correlation between the subjective intensity ratings and the BOLD signal $(r=0.28, d f=12, p=0.36 \mathrm{~ns})$; (e) shows the peak values $( \pm \operatorname{sem})$ of the $\%$ BOLD signal change at this site for the 4 thermal stimuli.

frontal cortex region were not strongly correlated with the intensity ratings as shown by no significant correlation in the SPM intensity correlation analysis, and by only a small correlation shown in Fig. $5 \mathrm{~d}$ $(r=0.65, p=0.016)$. To further elucidate the nature of the activation produced by the thermal stimuli in this region, Fig. 5e shows the peak values $( \pm$ sem $)$ of the $\%$ BOLD signal change at this site for the 4 thermal stimuli. It can be seen that the BOLD signals are graded in the order cold $>$ warm $1+$ cold $\approx$ warm $2+$ cold $>$ warm 2 , with activation to all the stimuli with a cold component, and deactivation to the warm2 stimulus.

In addition to this lateral orbitofrontal site, somewhat more anterior parts of the orbitofrontal cortex also had activations that were negatively correlated with the pleasantness ratings of the thermal stimuli [24 $54-8$ ] $z=3.16 p=0.016$ and [ $\left.\begin{array}{lll}-16 & 44 & -18\end{array}\right]$ $z=3.69 p=0.011$ (bilaterally); and also anteriorly there was a region with a positive correlation with intensity $\left[\begin{array}{lll}10 & 48 & -28\end{array}\right] z=3.42$ $p=0.016$.

Activations related to intensity but not to pleasantness in the somatosensory cortex

The SPM analysis showed a positive correlation with intensity in the somatosensory cortex with peak at $\left[\begin{array}{lll}-56 & -22 & 32\end{array}\right] z=4.03$ $p=0.001$ between the BOLD signal and the intensity ratings for the four thermal stimuli, as shown in Fig. 6a. The intensity ratings were clearly related to the BOLD signal, as shown in Fig. 6d $(r=0.78, p=0.002)$. Given that on average the stimuli were
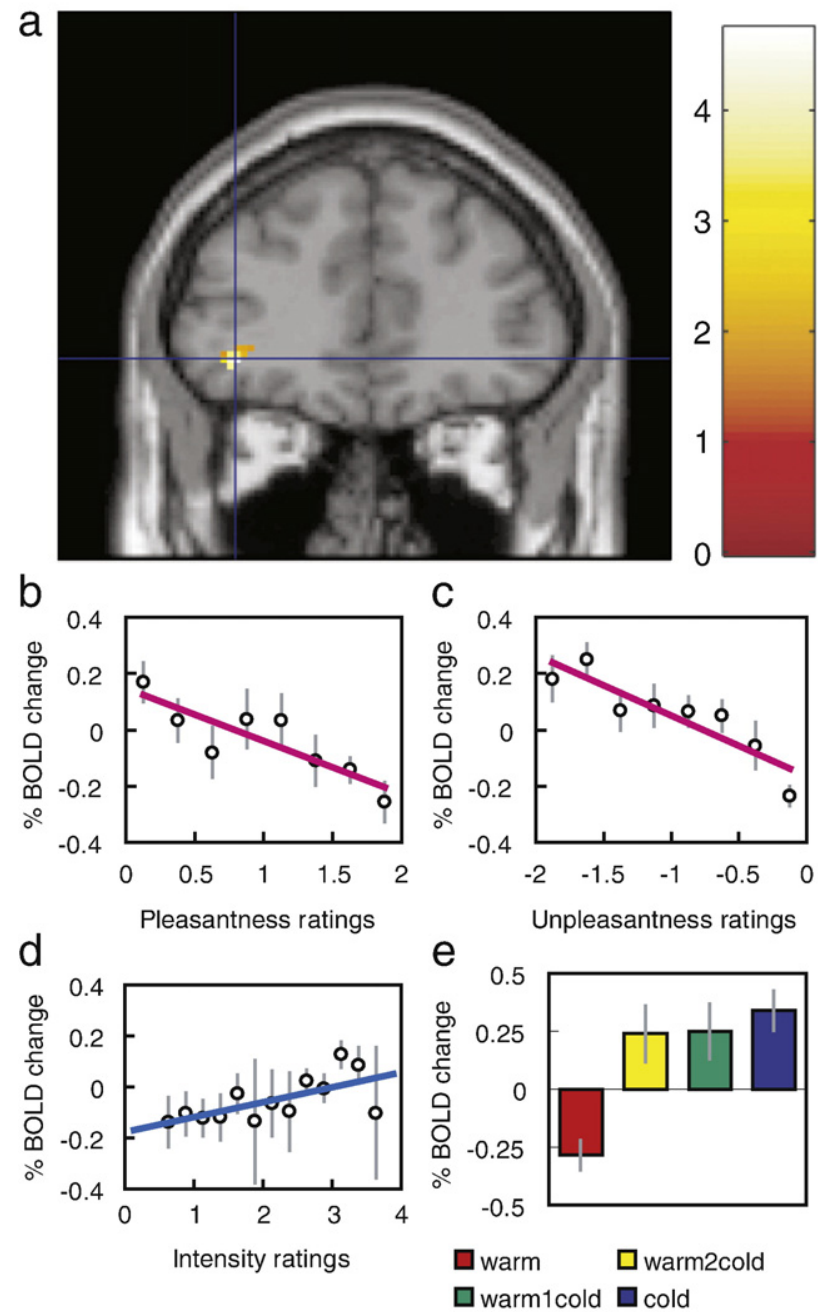

Fig. 5. Activations correlating with unpleasantness in the lateral orbitofrontal cortex. a. SPM analysis showing a negative correlation with pleasantness in the lateral orbitofrontal cortex with peak at $[-3840-6]$ between the BOLD signal and the pleasantness ratings of the four thermal stimuli. For this lateral orbitofrontal cortex region, (b) shows the negative correlation between the subjective pleasantness ratings for positive values of the ratings and the BOLD signal ( $r=-0.86, d f=7, p=0.006)$ (c) shows the negative correlation between the subjective pleasantness ratings for negative values of the ratings and the BOLD signal ( $r=-0.90, d f=7, p=0.003$ ); (d) shows that there is some correlation between the subjective intensity ratings and the BOLD signal $(r=0.65, d f=12, p=0.016)$; (e) shows the peak values $( \pm \mathrm{sem})$ of the $\%$ BOLD signal change at this site for the 4 thermal stimuli. 
approximately equally intense to the subjects (Fig. 1), the correlations with the intensity ratings are probably generated in part by the trial-by-trial variations in the perceived intensities and the related activations that occur. The SPM correlation analysis showed no significant correlation between the BOLD signal and the pleasantness ratings of the stimuli, and consistently there were no significant correlations with the pleasantness ratings for the data shown in Fig. $6 \mathrm{~b}(r=0.26, p=0.54)$ and $\mathrm{c}(r=-0.66, p=0.073)$. To further elucidate the nature of the activation produced by the thermal stimuli in this region, Fig. 6e shows the peak values $( \pm$ sem) of the \% BOLD signal change at this site for the four thermal stimuli. There is no graded activation according to the pleasantness of the stimuli, and instead there is somewhat greater
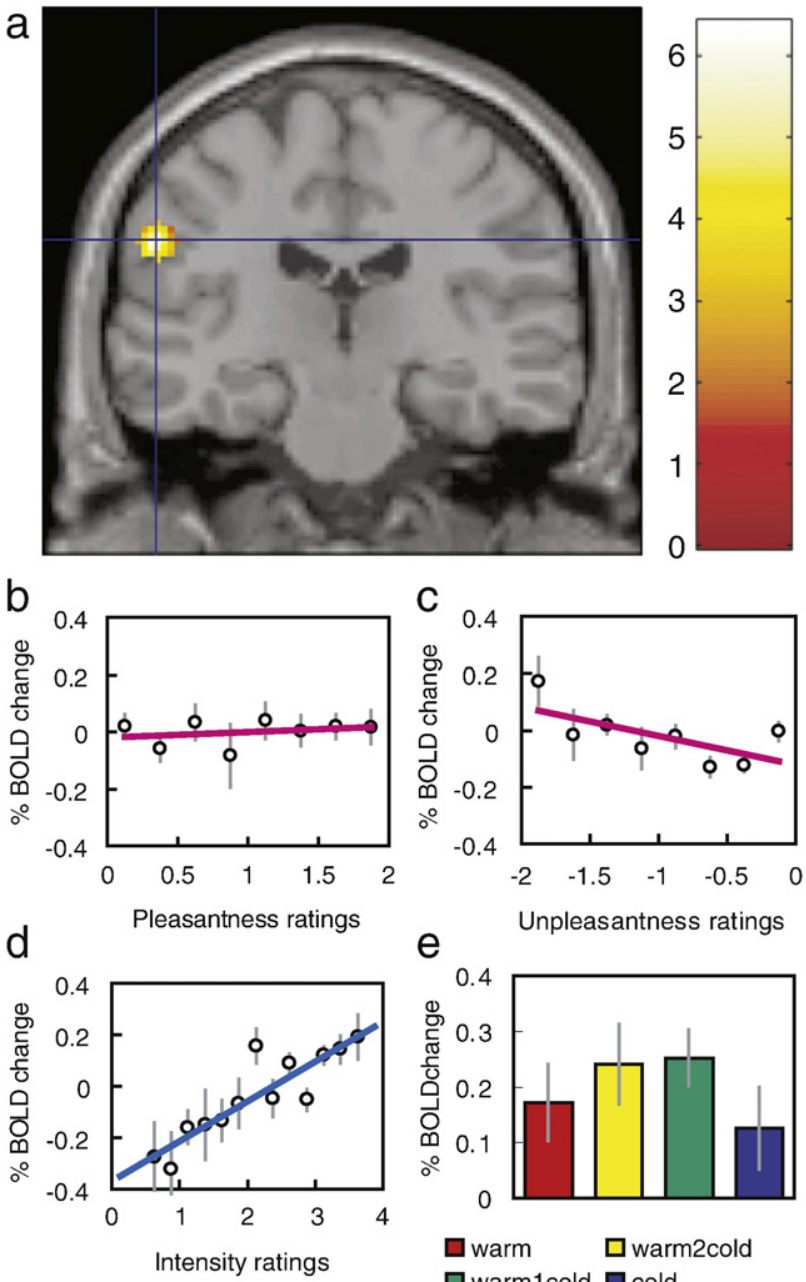

e

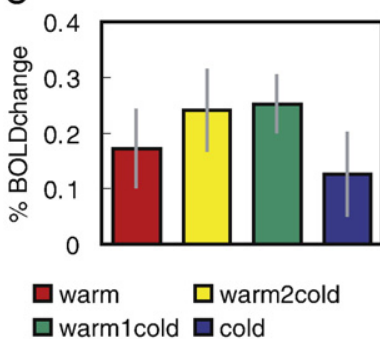

Fig. 6. Activations correlating with intensity and not with pleasantness in the somatosensory cortex. a. SPM analysis showing a correlation with intensity in the somatosensory cortex with peak at [-56-22 32] between the BOLD signal and the intensity ratings for the four thermal stimuli. For this somatosensory cortex region, (b) shows no correlation between the subjective pleasantness ratings for positive values of the ratings and the BOLD signal $(r=0.26, d f=7, p<0.54)$ (c) shows little correlation between the subjective pleasantness ratings for negative values of the ratings and the BOLD signal ( $r=-0.66, d f=7, p=0.073 \mathrm{~ns}$ ); (d) shows a positive correlation between the subjective intensity ratings and the BOLD signal ( $r=0.78, d f=12, p=0.002)$; (e) shows the peak values $( \pm \mathrm{sem})$ of the $\%$ BOLD signal change at this site for the 4 thermal stimuli.

\section{a}

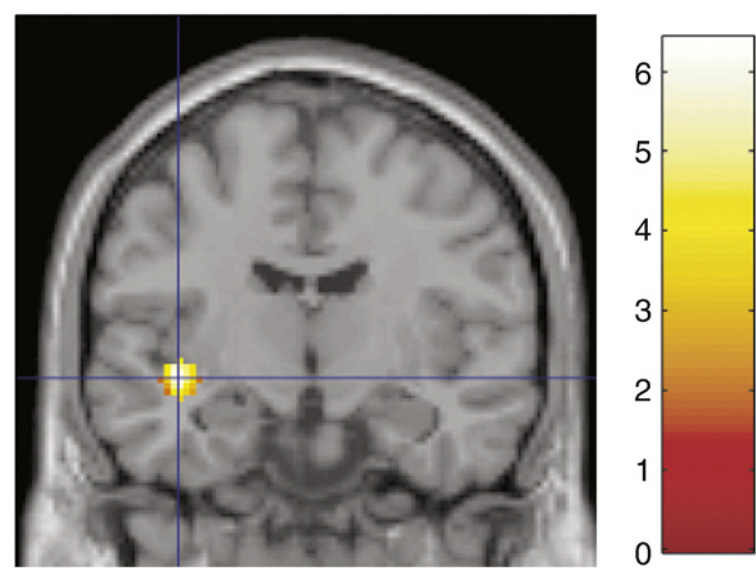

b

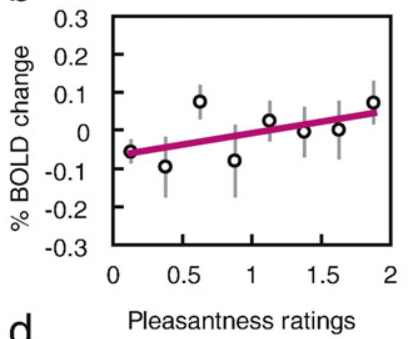

C
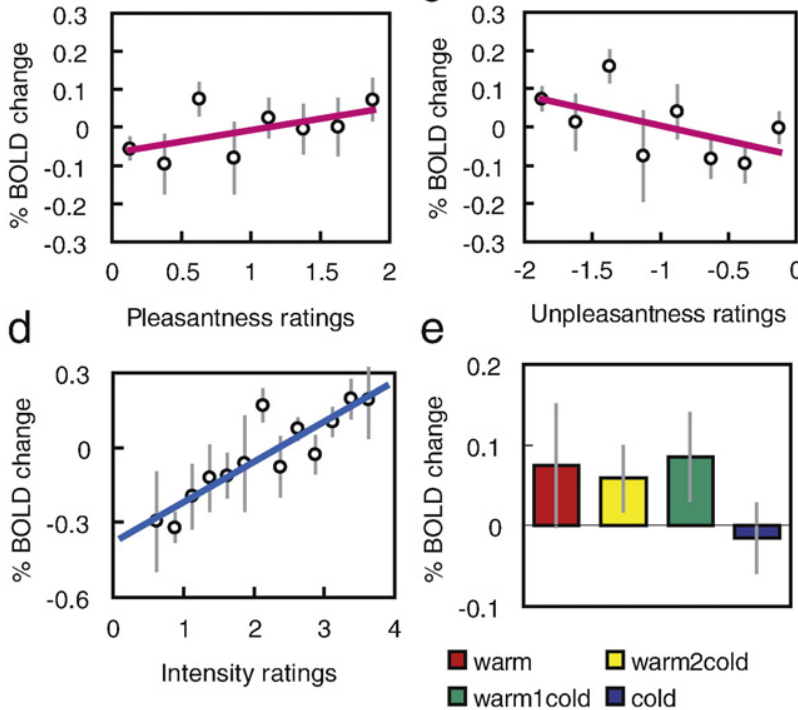

Fig. 7. Activations correlating with intensity and not with pleasantness in the posterior ventral insula. a. SPM analysis showing a correlation with intensity in the posterior ventral insula with peak at $[-40-10-8]$ between the BOLD signal and the intensity ratings for the four thermal stimuli. For this ventral insula cortex region, (b) shows no correlation between the subjective pleasantness ratings for positive values of the ratings and the BOLD signal ( $r=0.56, d f=7, p=0.15$ ) (c) shows no correlation between the subjective pleasantness ratings for negative values of the ratings and the BOLD signal ( $r=-0.56, d f=7, p=0.15)$; (d) shows a positive correlation between the subjective intensity ratings and the BOLD signal $(r=0.89, d f=12$, $p<0.001$ ); (e) shows the peak values ( \pm sem) of the \% BOLD signal change at this site for the 4 thermal stimuli.

activation to the mixed warm and cold stimuli than to the separate warm or cold stimuli. (The mixed stimuli have probably more afferent information, with both warm and cold being signalled, and this may account for the data in Fig. 6e.)

Activations related to intensity but not to pleasantness in the ventral insular cortex

The SPM analysis showed a positive correlation with intensity in the ventral insular cortex with peak at $[-40-10-8] z=4.05$ $p=0.001$ between the BOLD signal and the intensity ratings for 
the four thermal stimuli, as shown in Fig. 7a. The intensity ratings were clearly related to the BOLD signal, as shown in Fig. $7 \mathrm{~d}$ $(r=0.89, p<0.001)$. The SPM correlation analysis showed no significant correlation between the BOLD signal and the pleasantness ratings of the stimuli, and consistently there were no significant correlations with the pleasantness ratings for the data shown in Fig. $7 \mathrm{~b}(r=0.56, p=0.15)$ and $\mathrm{c}(r=-0.56, p=0.15)$. To further elucidate the nature of the activation produced by the thermal stimuli in this region, Fig. 7e shows the peak values $( \pm$ sem $)$ of the $\%$ BOLD signal change at this site for the 4 thermal stimuli. There is no graded activation according to the pleasantness of the stimuli.

The effect was bilateral, for additional peaks in the ventral insula where the activations correlated with intensity but not pleasantness were at [582 4] $z=3.66 p=0.003$; and in the anterior insula [42 10 - 12] $z=3.54 p=0.013$. (Consistently, in a PET study activations in both the mid-posterior insula and the anterior insula were correlated with intensity ratings of a set of cold stimuli (Craig et al., 2000)).

\section{Discussion}

The results provide interesting evidence for a separation into different brain systems where activations are related to the pleasantness, the unpleasantness, or the intensity of thermal stimuli.

Let us consider the areas where the activations were correlated with the intensity and not the pleasantness ratings to these innocuous thermal stimuli. These areas included the ventral posterior insular cortex and a part of the somatosensory cortex. The implication is that the temperature being applied to the skin is represented in these areas, but not in a way where overall the neuronal activation increases in the order of the hedonic value of the stimuli. Indeed, different neurons in these areas may be broadly tuned to temperatures, or may have increasing or decreasing firing as a function of temperature, which is what we have found for oral thermosensitive neurons in the insular cortex (Verhagen et al., 2004). Such a representation conveys much information about the temperature being represented, especially if each neuron is not too specifically tuned to a particular temperature, so that the benefits of a distributed representation are obtained (Rolls, 2008b). The implication of the results described here is that this type of representation may be present in these areas. Although the activations in these regions were correlated with the intensities of the stimuli, the stimuli were not rated on average across the whole population of subjects as being significantly differently intense. The correlations with subjective intensity thus may arise because different subjects rate the intensity of the stimuli differently but nevertheless as individual subjects have brain areas where the activations correlate with the intensity, or because of trial-by-trial variation in the rated intensity and the BOLD signal. Consistent with the effects described here, non-noxious thermal stimuli have been shown to activate the insular cortex (Casey et al., 1996; Craig et al., 2000; Guest et al., 2007; Hua le et al., 2005); and stroke-induced lesions of the insular cortex can produce selective loss of non-painful thermal sensation (Cattaneo et al., 2007).

Parts of the orbitofrontal cortex (e.g. mid-orbitofrontal cortex, Fig. 2) and pregenual cingulate cortex (Fig. 3) had activations that were not only correlated with the pleasantness of the thermal stimuli, but were also graded, with the warm 2 stimulus producing most activation, the cold stimulus most deactivation, and the intermediate combination stimuli intermediate activations. At these sites, correlations with intensity were absent or low. The implication is that the neuronal activation in these regions is on average graded as a function of increasing pleasantness. In turn, this means that the pleasantness is made explicit in the representation, in that a decoder as simple as a neuron that summates inputs from these regions provides a useful estimate of the pleasantness of the stimuli, which can then be used to influence subsequent behavior, for example, of whether that stimulus should be a goal for action, or a decision made to obtain that stimulus (Rolls, 2005, 2008b).

The findings on these orbitofrontal and pregenual cingulate regions are consistent, and indeed provide strong support for, evidence that the pleasantness of a number of other stimuli is represented in these regions, including pleasant touch (McCabe et al., 2008; Rolls, in press, 2003b), pleasant odor (Grabenhorst et al., 2007b; Rolls et al., 2008b, 2003a), pleasant flavour (McCabe and Rolls, 2007; Rolls and McCabe, 2007), pleasant taste (Grabenhorst and Rolls, 2008), winning money (O'Doherty et al., 2001a), and the attractiveness of a face (O'Doherty et al., 2003) (see also Kringelbach and Rolls, 2004; Rolls, 2005, 2008a,b). Does this mean that the representation in these regions is of something general, such as general pleasantness? Although the functional imaging evidence does not address this very directly, the evidence from single neuron recording in macaques does provide a very clear answer, with (oral) temperature orbitofrontal cortex neurons responding in some cases only to temperature, and in other cases to temperature and/or capsaicin and/or taste and/or viscosity and/or gritty texture and/or fat texture (Kadohisa et al., 2004). Thus the representation at the neuronal level in the orbitofrontal cortex is of the specific details of each sensory stimulus (Rolls, 2005, 2006), but in a way in which the hedonics is made explicit in the representation, in that for example some neurons decrease their response to a food stimulus when that food is fed to satiety (Rolls et al., 1989). Information about skin temperature may reach the orbitofrontal cortex from the somatosensory cortex and insular somatosensory areas, which project to the orbitofrontal cortex (Carmichael and Price, 1995).

It was of interest that activations in some parts of the ventral striatum are also correlated with the pleasantness of the thermal stimuli, and it is known that the orbitofrontal cortex sends connections to the ventral striatum (Ferry et al., 2000) providing a possible route for information of the type encoded in the orbitofrontal cortex to reach the ventral striatum. The ventral striatum may be similarly influenced by other reward representations in the orbitofrontal cortex (Grabenhorst et al., 2007a; O'Doherty et al., 2002; Rolls and McCabe, 2007; Rolls et al., 2008a), and may play a role in the incentive value of reward and punishment-related stimuli (Cardinal et al., 2002; Everitt and Robbins, 2005).

Parts of the orbitofrontal cortex (e.g. lateral orbitofrontal cortex, Fig. 5) had activations that were correlated with the unpleasantness of the thermal stimuli, but were also graded, with the cold stimulus producing most activation, the warm 2 stimulus most deactivation, and the intermediate combination stimuli intermediate activations. At this site, the correlation with intensity is relatively low. The implication is that in this area the unpleasantness of thermal stimuli is made explicit in the representation. The unpleasantness of a number of other stimuli is represented in this region, including unpleasant touch (Rolls et al., 2003b), unpleasant odor (Rolls et al., 2003a), and losing money (Kringelbach and Rolls, 2004; Rolls, 2008a).

In control analyses, similar results to those described above were found when the analyses used only the trials on which both warm and cold stimuli were presented (warm $2+$ cold and warm $1+$ cold), showing that the results were related to the pleasantness and 
intensity produced by the stimuli, and not to the site where they were applied (dorsum vs palm of the hand), or to the time taken to reach the steady state, as these factors were the same for this subset of stimuli (see Supplementary Material). These control analyses were helpful as they showed that, for example, the rise times of the stimuli, or their spatial location, were not confounding factors to the results described. We further note that the correlations we described were with the pleasantness ratings of the thermal stimuli, and that such correlations cannot be in the general case with the absolute value of the temperature, as can be seen by the fact that if we had increased the temperature to the noxious range with a "hot" stimulus of perhaps $45^{\circ} \mathrm{C}$, then such a stimulus would break any correlation at a pleasantness site with the absolute value of the temperature. We further note that the fMRI results confirm the efficacy of obtaining two independent ratings from participants, one of intensity, and another for affective value, for the activations in different brain areas correlated with these different ratings.

The findings show that activations in brain regions such as the orbitofrontal and pregenual cingulate cortex and ventral striatum are correlated with the affective experience or feeling reported by the participants in the ratings they made. We thus suggest that processing in these brain regions is related to the affective feelings that are reported. This does not necessarily mean that the subjective feelings are actually represented in these brain areas, as these brain areas could have onward connections to further brain areas where the feelings themselves might be generated (Rolls, 2005, 2007, 2008b). Consistent with the importance of some of these areas in the feelings that do arise from thermal stimulation, strokes that damage the insula may lead to selective loss of non-painful and painful thermal sensation (Birklein et al., 2005; Cattaneo et al., 2007). However, the important principle revealed in this investigation is that the cortical areas that perform the processing that is related to the affective value of thermal stimuli are different from those related to the sensory properties of the stimuli such as their intensity, with our study showing that affective value is related to activations in the orbitofrontal cortex and pregenual cingulate cortex, and (consistent with PET data (Craig et al., 2000)) that activations in the insula are correlated with ratings of the intensity of the thermal stimuli. Part of the importance of this is that it is important to be able to represent the pleasantness of a thermal stimulus, which may direct behaviour towards or away from it, separately from the fact that it is warm or cold, and its intensity, which may be important to remember for future use independently of its current affective value. Psychophysically, the current affective value of a thermal stimulus, but not the perception of its warmness or coolness, is dependent on core body temperature (Cabanac, 1971; Mower, 1976). For comparison, and consistently, in the primary olfactory areas such as the pyriform cortex, the subjective intensity ratings but not the pleasantness ratings are correlated with the activations (Anderson et al., 2003; Grabenhorst et al., 2007b; Rolls et al., 2003a), and analogous findings are available for taste (Grabenhorst et al., 2007a). It is an interesting issue about why these types of processing are segregated in the brain. One possible reason is that it may be important to have a representation of what a stimulus is that is independent of its current value, so that if the stimulus is encountered when it is of no value, as may be the case for example for a warm stimulus if our body temperature is high (Cabanac, 1971; Mower, 1976), then nevertheless we can represent what the stimulus is, learn where it is in the environment for future use, etc (Rolls, 2005, 2008b). A second possible advantage of segregated representations of affective value and the physical properties of stimuli such as their intensity is that when a computation is being performed about affective value, it may be useful to have all the representations of the values of different stimuli represented close in the brain, so that by interaction implemented for example by lateral inhibition the currently most rewarding stimulus may be easily computed. The evolutionary advantage arises because the neurons involved in this type of computation need to communicate with each other, and not with neurons representing for example the full raw sensory details of every stimulus, and the wiring length of the neurons that need to communicate is minimized by placing them close together in the brain, thus helping to minimize brain size (Cowey, 1979; Rolls, 2008b; Rolls and Deco, 2002). In comparison, the processing of the physical properties of taste, smell, touch, temperature, etc each takes place in its own early cortical areas, where activations are related to physical properties of the stimuli such as their intensity, and this is consistent with the overall concept, for processing of the physical properties of taste, touch, sight, smell etc can be performed relatively independently in early stage cortical processing. In contrast, affective processing for different sensory modalities can perhaps best be performed in common areas such as the orbitofrontal and pregenual cingulate cortex, where the reward values of the different stimuli can be directly compared.

\section{Acknowledgments}

F.G. was supported by the Gottlieb-Daimler- and Karl BenzFoundation. The investigation was performed at the Centre for Functional Magnetic Resonance Imaging of the Brain (FMRIB) at Oxford University, and we thank Peter Hobden, Siri Leknes, Katie Warnaby, and Irene Tracey for their help.

\section{Appendix A. Supplementary data}

Supplementary data associated with this article can be found, in the online version, at doi:10.1016/j.neuroimage.2008.03.005.

\section{References}

Anderson, A.K., Christoff, K., Stappen, I., Panitz, D., Ghahremani, D.G., Glover, G., Gabrieli, J.D., Sobel, N., 2003. Dissociated neural representations of intensity and valence in human olfaction. Nat. Neurosci. 6 , 196-202.

Bantick, S.J., Wise, R.G., Ploghaus, A., Clare, S., Smith, S.M., Tracey, I., 2002. Imaging how attention modulates pain in humans using functional MRI. Brain 125, 310-319.

Becerra, L., Breiter, H.C., Wise, R., Gonzalez, R.G., Borsook, D., 2001. Reward circuitry activation by noxious thermal stimuli. Neuron 32, 927-946.

Birklein, F., Rolke, R., Muller-Forell, W., 2005. Isolated insular infarction eliminates contralateral cold, cold pain, and pinprick perception. Neurology 65, 1381.

Brooks, J.C., Zambreanu, L., Godinez, A., Craig, A.D., Tracey, I., 2005. Somatotopic organisation of the human insula to painful heat studied with high resolution functional imaging. NeuroImage 27, 201-209.

Cabanac, M., 1971. Physiological role of pleasure. Science 173, 1103-1107. Cabanac, M., 2002. What is emotion? Behav Processes 60, 69-83.

Cardinal, N., Parkinson, J.A., Hall, J., Everitt, B.J., 2002. Emotion and motivation: the role of the amygdala, ventral striatum, and prefrontal cortex. Neurosci. Biobehav. Rev. 26, 321-352.

Carmichael, S.T., Price, J.L., 1995. Sensory and premotor connections of the orbital and medial prefrontal cortex of macaque monkeys. J. Comp. Neurol. 363, 642-664. 
Casey, K.L., Minoshima, S., Morrow, T.J., Koeppe, R.A., 1996. Comparison of human cerebral activation pattern during cutaneous warmth, heat pain, and deep cold pain. J. Neurophysiol. 76, 571-581.

Cattaneo, L., Chierici, E., Cucurachi, L., Cobelli, R., Pavesi, G., 2007. Posterior insular stroke causing selective loss of contralateral nonpainful thermal sensation. Neurology 68, 237.

Collins, D.L., Neelin, P., Peters, T.M., Evans, A.C., 1994. Automatic 3D intersubject registration of MR volumetric data in standardized Talairach space. J. Comput. Assist. Tomogr. 18, 192-205.

Cowey, A., 1979. Cortical maps and visual perception: the Grindley Memorial Lecture. Q. J. Exp. Psychol. 31, 1-17.

Craig, A.D., Reiman, E.M., Evans, A., Bushnell, M.C., 1996. Functional imaging of an illusion of pain. Nature 384, 258-260.

Craig, A.D., Chen, K., Bandy, D., Reiman, E.M., 2000. Thermosensory activation of insular cortex. Nat. Neurosci. 3, 184-190.

de Araujo, I.E.T., Kringelbach, M.L., Rolls, E.T., Hobden, P., 2003. The representation of umami taste in the human brain. J. Neurophysiol. 90, 313-319.

de Araujo, I.E.T., Rolls, E.T., Velazco, M.I., Margot, C., Cayeux, I., 2005. Cognitive modulation of olfactory processing. Neuron 46, 671-679.

Everitt, B.J., Robbins, T.W., 2005. Neural systems of reinforcement for drug addiction: from actions to habits to compulsion. Nat. Neurosci. 8, 1481-1489.

Ferry, A.T., Ongur, D., An, X., Price, J.L., 2000. Prefrontal cortical projections to the striatum in macaque monkeys: evidence for an organization related to prefrontal networks. J. Comp. Neurol. 425, 447-470.

Friston, K.J., Glaser, D.E., Henson, R.N., Kiebel, S., Phillips, C., Ashburner, J., 2002. Classical and Bayesian inference in neuroimaging: applications. NeuroImage 16, 484-512.

Friston, K.J., Worsley, K.J., Frackowiak, R.S.J., Mazziotta, J.C., Evans, A.C., 1994. Assessing the significance of focal activations using their spatial extent. Hum. Brain Mapp. 1, 214-220.

Grabenhorst, F., Rolls, E.T., 2008. Selective attention to affective value alters how the brain processes taste stimuli. Eur. J. Neurosci. 27, 723-729.

Grabenhorst, F., Rolls, E.T., Bilderbeck, A., 2007a. How cognition modulates affective responses to taste and flavor: top down influences on the orbitofrontal and pregenual cingulate cortices. Cereb. Cortex. doi:10.1093/cercor/bhm185.

Grabenhorst, F., Rolls, E.T., Margot, C., da Silva, M.A.A.P., Velazco, M.I., 2007b. How pleasant and unpleasant stimuli combine in different brain regions: odor mixtures. J. Neurosci. 27, 13532-13540.

Guest, S., Grabenhorst, F., Essick, G., Chen, Y., Young, M., McGlone, F., de Araujo, I., Rolls, E.T., 2007. Human cortical representation of oral temperature. Physiol. Behav. 92, 975-984.

Hua le, H., Strigo, I.A., Baxter, L.C., Johnson, S.C., Craig, A.D., 2005. Anteroposterior somatotopy of innocuous cooling activation focus in human dorsal posterior insular cortex. Am. J. Physiol. Regul. Integr. Comp. Physiol. 289, R319-R325.

Kadohisa, M., Rolls, E.T., Verhagen, J.V., 2004. Orbitofrontal cortex neuronal representation of temperature and capsaicin in the mouth. Neuroscience 127, 207-221.

Kadohisa, M., Rolls, E.T., Verhagen, J.V., 2005a. Neuronal representations of stimuli in the mouth: the primate insular taste cortex, orbitofrontal cortex, and amygdala. Chem. Senses 30, 401-419.

Kadohisa, M., Rolls, E.T., Verhagen, J.V., 2005b. The primate amygdala: neuronal representations of the viscosity, fat texture, temperature, grittiness and taste of foods. Neuroscience 132, 33-48.

Kiebel, S.J., Poline, J.B., Friston, K.J., Holmes, A.P., Worsley, K.J., 1999. Robust smoothness estimation in statistical parametric maps using standardized residuals from the general linear model. Neurolmage. 10, 756-766.

Kringelbach, M.L., Rolls, E.T., 2004. The functional neuroanatomy of the human orbitofrontal cortex: evidence from neuroimaging and neuropsychology. Prog. Neurobiol. 72, 341-372.

McCabe, C., Rolls, E.T., 2007. Umami: a delicious flavor formed by convergence of taste and olfactory pathways in the human brain. Eur. J. Neurosci. 25, 1855-1864.
McCabe, C., Rolls, E.T., Bilderbeck, A., McGlone, F., 2008. Cognitive influences on the representation of touch in the human brain. Social, Cognitive and Affective Neuroscience 3. doi:10.1093/scan/nsn005.

Mower, G.D., 1976. Perceived intensity of peripheral thermal stimuli is independent of internal body temperature. J. Comp. Physiol. Psychol. 90, 1152-1155.

O’Doherty, J., Kringelbach, M.L., Rolls, E.T., Hornak, J., Andrews, C., 2001a. Abstract reward and punishment representations in the human orbitofrontal cortex. Nat. Neurosci. 4, 95-102.

O’Doherty, J., Rolls, E.T., Francis, S., Bowtell, R., McGlone, F., 2001b. The representation of pleasant and aversive taste in the human brain. J. Neurophysiol. 85, 1315-1321.

O’Doherty, J.P., Deichmann, R., Critchley, H.D., Dolan, R.J., 2002. Neural responses during anticipation of a primary taste reward. Neuron 33, 815-826.

O’Doherty, J., Winston, J., Critchley, H., Perrett, D., Burt, D.M., Dolan, R.J., 2003. Beauty in a smile: the role of medial orbitofrontal cortex in facial attractiveness. Neuropsychologia 41, 147-155.

Rolls, E.T., 1999. The Brain and Emotion. Oxford University Press, Oxford. Rolls, E.T., 2005. Emotion Explained. Oxford University Press, Oxford.

Rolls, E.T., 2006. Brain mechanisms underlying flavour and appetite. Philos. Trans. R. Soc. Lond., B 361, 1123-1136.

Rolls, E.T., 2007. A computational neuroscience approach to consciousness. Neural Netw. 20, 962-982.

Rolls, E.T., in press. The affective and cognitive processing of touch, oral texture, and temperature in the brain. Neurosci. Biobehav. Rev. doi:10.1016/j.neubiorev.2008.03.010.

Rolls, E.T., 2008a. The anterior and midcingulate cortices and reward. In: Vogt, B.A. (Ed.), Cingulate Neurobiology and Disease. Oxford University Press, Oxford.

Rolls, E.T., 2008b. Memory, Attention, and Decision-Making: a Unifying Computational Neuroscience Approach. Oxford University Press, Oxford.

Rolls, E.T., Deco, G., 2002. Computational Neuroscience of Vision. Oxford University Press, Oxford.

Rolls, E.T., McCabe, C., 2007. Enhanced affective brain representations of chocolate in cravers vs non-cravers. Eur. J. Neurosci. 26, 1067-1076.

Rolls, E.T., Sienkiewicz, Z.J., Yaxley, S., 1989. Hunger modulates the responses to gustatory stimuli of single neurons in the caudolateral orbitofrontal cortex of the macaque monkey. Euro. J. Neurosci. 1, 53-60.

Rolls, E.T., Kringelbach, M.L., de Araujo, I.E.T., 2003a. Different representations of pleasant and unpleasant odors in the human brain. Eur. J. Neurosci. 18, 695-703.

Rolls, E.T., O’Doherty, J., Kringelbach, M.L., Francis, S., Bowtell, R., McGlone, F., 2003b. Representations of pleasant and painful touch in the human orbitofrontal and cingulate cortices. Cerebral Cortex 13, 308-317.

Rolls, E.T., McCabe, C., Redoute, J., 2008a. Expected value, reward outcome, and temporal difference error representations in a probabilistic decision task. Cereb. Cortex 18, 652-663.

Rolls, E.T., Grabenhorst, F., Margot, C., da Silva, M.A.A.P., Velazco, M.I., 2008b. Selective attention to affective value alters how the brain processes olfactory stimuli. J. Cogn. Neurosci. 20. (Electronic publication ahead of print 27 March).

Tracey, I., Becerra, L., Chang, I., Breiter, H., Jenkins, L., Borsook, D., Gonzalez, R.G., 2000. Noxious hot and cold stimulation produce common patterns of brain activation in humans: a functional magnetic resonance imaging study. Neurosci Lett. 288, 159-162.

Verhagen, J.V., Kadohisa, M., Rolls, E.T., 2004. The primate insular/opercular taste cortex: neuronal representations of the viscosity, fat texture, grittiness, temperature and taste of foods. J. Neurophysiol. 92, 1685-1699.

Wilson, J.L., Jenkinson, M., Araujo, I.E.T., Kringelbach, M.L., Rolls, E.T., Jezzard, P., 2002. Fast, fully automated global and local magnetic field optimisation for fMRI of the human brain. NeuroImage 17, 967-976.

Worsley, K.J., Marrett, P., Neelin, A.C., Friston, K.J., Evans, A.C., 1996. A unified statistical approach for determining significant signals in images of cerebral activation. Hum. Brain Mapp. 4, 58-73. 